\title{
Rare Case of Left Ventricular Thrombus Postmyocardial Infarction for Emergency Decompressive Craniectomy
}

\author{
Thirumurugan Arikrishnan ${ }^{1}$ \\ Deepak Chakravarthy ${ }^{10}$ \\ Duraiyarassu Uthaman ${ }^{1}$ \\ Gnanasekaran Srinivasan ${ }^{1}$
}

\author{
${ }^{1}$ Department of Anaesthesiology and Critical Care, Jawaharlal \\ Institute of Postgraduate Medical Education and Research, \\ Puducherry, India
}

J Neuroanaesthesiol Crit Care 2022;9:112-114.
Address for correspondence Thirumurugan Arikrishnan, MD, Department of Anaesthesiology and Critical Care, Jawaharlal Institute of Postgraduate Medical Education and Research, Dhanvantri Nagar, Puducherry 605006, India (e-mail: a.thiru1990t@gmail.com).

\begin{abstract}
Keywords

- craniectomy

- left ventricular thrombus

- myocardial infarction

Left ventricular (LV) thrombus formation is a notorious complication encountered in postmyocardial infarction patients. Such cases, when coming for noncardiac surgery, put the patient at greater risk of embolic events. Anesthesiologists play a pivotal role in the management of such rare and difficult cases. There is sparse evidence on management of such cases for noncardiac surgery. Hence, we would like to share our experience of a young patient with LV thrombus posted for left decompressive craniectomy.
\end{abstract}

\section{Introduction}

Left ventricular (LV) thrombus is not an uncommon complication encountered after myocardial infarction(MI). The incidence of $\mathrm{LV}$ thrombus is $15 \%$ with anterior wall MI (AWMI). ${ }^{1}$ The presence of residual clot along with embolic complications pose significant challenges to an anesthesiologist in terms of managing not only the surgical procedure addressing the embolic complication but also the underlying cardiac condition that resulted in clot formation, more often MI or dilated cardiomyopathy. We present a case of post-MI LV clot with thromboembolic stroke posted for decompressive craniectomy.

\section{Case Report}

A 36-year-old male presented with complaints of weakness of right upper and lower limbs, associated with opposite side facial deviation and slurring of speech for 5 days. He was diagnosed to have AWMI 4 months before presentation.
Thrombolysis was done with 1.5 million units of streptokinase intravenously (IV). History revealed noncompliance to anticoagulant medication. He then developed left middle cerebral artery (MCA) infarct ( - Fig. 1). Examination revealed right hemiplegia with $0 / 5$ power, right upper motor neuron facial palsy, and Glasgow coma scale (GCS) score of 14/15. ECG revealed Q waves in leads 1 and aVL. Echocardiography showed severe hypokinesia of anterior, anteroseptal, and apical walls with $20 \%$ ejection fraction (EF) and large LV thrombus of size $5.3 \times 4.3 \mathrm{~cm}$, extending from apex to midventricular cavity. Coronary angiogram postthrombolysis revealed recanalized left anterior descending (LAD) artery. Two days later, emergency left decompressive craniectomy was planned in view of neurological deterioration and increased midline shift despite medical management. Preinduction arterial line was secured. Opioid-based induction was done with IV fentanyl $150 \mathrm{mcg}$, $2 \%$ sevoflurane, IV rocuronium $50 \mathrm{mg}$; IV lignocaine $80 \mathrm{mg}$ was given to attenuate intubation response. Airway was secured with published online October 24, 2021
DOI https://doi.org/ 10.1055/s-0041-1734421. ISSN 2348-0548.

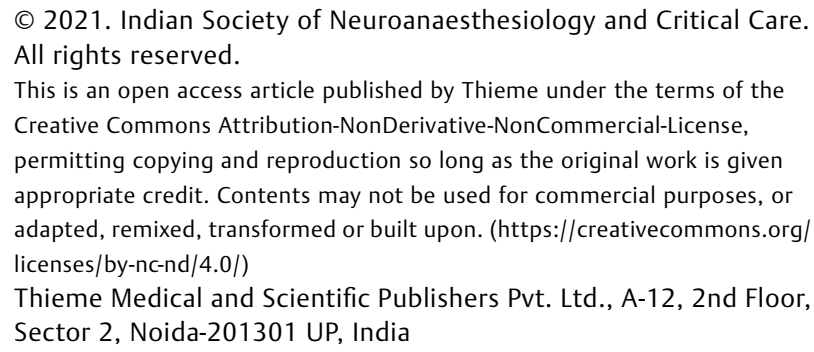

(C) 2021. Indian Society of Neuroanaesthesiology and Critical Care. All rights reserved.

This is an open access article published by Thieme under the terms of the Creative Commons Attribution-NonDerivative-NonCommercial-License, permitting copying and reproduction so long as the original work is given appropriate credit. Contents may not be used for commercial purposes, or adapted, remixed, transformed or built upon. (https://creativecommons.org/ licenses/by-nc-nd/4.0/)

Thieme Medical and Scientific Publishers Pvt. Ltd., A-12, 2nd Floor, Sector 2, Noida-201301 UP, India 


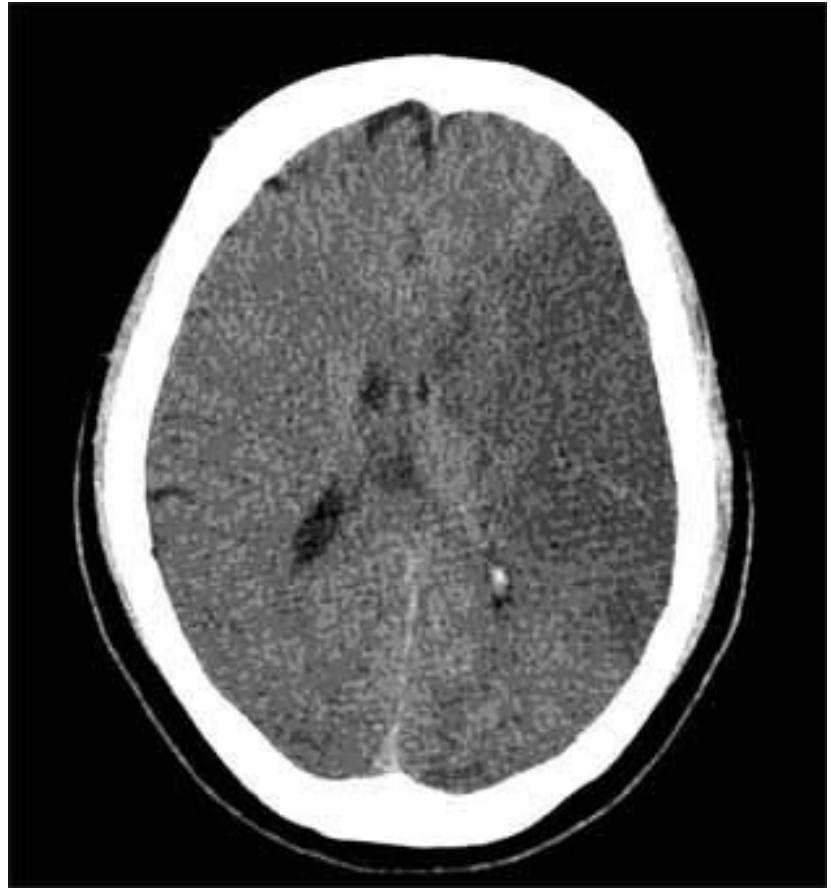

Fig. 1 Preoperative noncontrast CT brain image showing left middle cerebral artery (MCA) infarct.

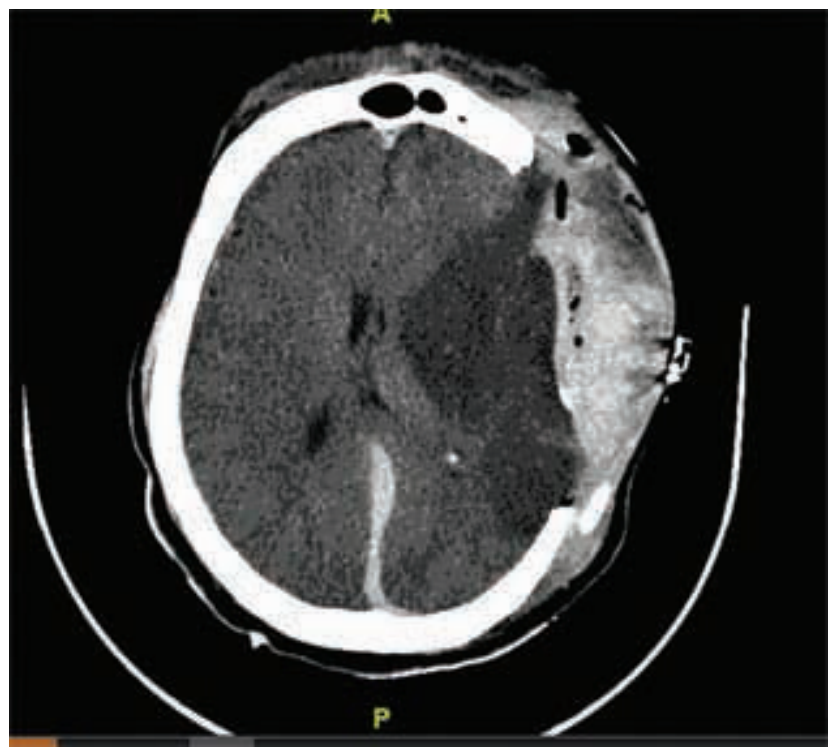

Fig. 2 Noncontrast CT brain image showing status postdecompressive craniectomy.

endotracheal intubation. Right subclavian central venous catheter was secured. To prevent hemodynamic response to surgical incision, scalp block was given with $20 \mathrm{~mL}$ of $0.25 \%$ bupivacaine. Anesthesia was maintained with isoflurane in air-oxygen mixture. Hypertonic saline and furosemide were administered as antiedema measures. Surgery lasted for 2 hours and the patient was not extubated in view of diffuse brain bulge and was electively ventilated (-Fig. 2 ). The patient was restarted on cardiac medications and enoxaparin $60 \mathrm{mg}$ OD from postoperative day 1 (POD-1) and was extubated with full GCS score on POD-2. Enoxaparin dosage was stepped up to BD dosage on POD-3 and later switched over to clopidogrel on POD-5.

\section{Discussion}

LV thrombus formation occurs due to presence of Virchow's triad; reduced contractility and increased LV internal diameter leads to stasis, local myocardial injury (endothelial injury), and presence of proinflammatory or procoagulable state. Determining factors for clot formation include degree of myocardial injury, ST-segment elevation myocardial infarction (STEMI) more than non-ST-segment elevation myocardial infarction (NSTEMI), apicoanterior wall lesions more than others, and EF less than $40 \%{ }^{2}$ The risk of embolization is uncertain, but it can be up to 10 to $20 \%$ in the first 3 months after MI and thereafter risk decreases as the thrombus becomes organized. ${ }^{1}$ Mobile pedunculated clots are associated with increased risk of embolization. ${ }^{3}$ LV clot accounts for $10 \%$ cases of cardioembolic stroke. ${ }^{4}$ Our patient had risk factors associated with LV thrombus formation like previous AWMI, low EF, and noncompliance to systemic anticoagulation. Although he had an organized clot, and the inciting MI event had occurred more than 3 months ago, he still developed cardioembolic stroke due to refraining from anticoagulation.

Intraoperative plan was to maintain preload, avoid tachycardia, maintain systemic vascular resistance (SVR), avoid hypotension, and maintain a normal sinus rhythm. Sevoflurane has little effect on cardiac output and SVR in graduated doses compared with IV induction agents. ${ }^{5}$ Hence, a combination of opioid-based induction with sevoflurane was used for induction. An IV bolus of lignocaine effectively attenuated the pressor response of tracheal intubation. Surgeons were advised to avoid adrenaline infiltration to prevent tachycardia. Surgical response was well blunted by scalp block, thereby allowing us to use lesser anesthetic concentrations at 0.5 minimum alveolar concentration (MAC), which was beneficial both for negating the cardiodepressant action on the poorly contracting heart and avoiding increase in cerebral blood flow by inhalational anesthetics. The general principle to avoid clot embolization revolved around avoiding a hyperdynamic heart and avoiding tachycardia, arrhythmias and increased contractility, and maintaining perfusion without the use of inotropes. Inotropes, by increasing contractility, can theoretically cause embolization. If the dislodged embolus size is big enough to cause LV outflow tract obstruction, then on-table cardiac arrest is inevitable, and cardiopulmonary resuscitation (CPR) and defibrillation increases the risk of further embolization. So, the case was operated under cardiopulmonary bypass ( $\mathrm{CPB}$ ) backup in case of any untoward events as a resuscitative measure, and measures to minimize the chances of clot dislodgement were taken. Intraoperatively, dynamic fluid responsiveness parameters were used to administer fluids and blood. Since the space inside LV cavity other than thrombus is small, despite dilatation of the ventricles, patient cannot tolerate hypovolemia or hypervolemia. Hence, maintaining adequate volume is of paramount importance. To reduce intracranial 
pressure, furosemide and hypertonic saline were used. Mannitol was avoided, as it can precipitate cardiac failure in the setting of poor cardiac function. Postoperatively, enoxaparin $60 \mathrm{mg}$ subcutaneously (SC) was started on POD-1 as once daily dose and stepped up to $60 \mathrm{mg}$ SC twice daily dosage to prevent recurrent systemic embolization. Anticoagulation was increased gradually to avoid the risk of intracranial bleed from recent surgery and to allow adequate time for surveillance for bleeding. Although the possibility of concomitant surgical thrombectomy and decompressive craniectomy could have been ideal, it was not practically possible as LV thrombectomy would have required anticoagulation and risked intracranial bleeding. Moreover, LV thrombectomy is usually allocated to patients with high risk of embolization like prior embolic episodes, floating thrombus, and unsuccessful anticoagulation therapy. This procedure usually requires $\mathrm{LV}$ incision which, in turn, can lead to further $\mathrm{LV}$ dysfunction, arrhythmias, and aneurysm formation. Hence, we did not combine LV thrombectomy and decompressive craniectomy in the same sitting.

Such cases coming for noncardiac surgery in an emergency setting are rare and difficult to manage due to complexity in decision-making and also due to their complex cardioneurological hemodynamic interplays. Although speculations that $\mathrm{LV}$ thrombus plays a positive role in the acutely damaged myocardium by offering mechanical support to the infarcted areas by reducing myocardial bulge and preventing rupture resulting in effective myocardial contraction have been proposed, ${ }^{6} \mathrm{LV}$ clot presents as "sword of the Damocles," producing an imminent everlasting risk of systemic embolism.

\section{Conflict of Interest}

None declared.

\section{References}

1 Osman I, Le D, Movahed A, et al. A fatal giant left ventricular thrombus protruding to the left atrium after anterior wall myocardial infarction. Heart Circ 2017; 1:14

2 Habash F, Vallurupalli S. Challenges in management of left ventricular thrombus. Ther Adv Cardiovasc Dis 2017;11(08): 203-213

3 Madhusudhana R, Kumar R, Dinesh K, Somasekharam P. A case of patient with left ventricular clot posted for laparotomy. KAJ 2010; 11:40-42

4 Taneja R, Aguirre MA, Shi C, Greilich PE. Embolization of left ventricular apical thrombus during cardiac surgery. Anesth Analg 2010;111(01):74-75

5 Kokawa K, Iranami H, Maeda H, Tajima T, Yamamoto M, Hatano Y. Sevoflurane anesthesia in patients with cardiomyopathy. JJpn Soc Clin Anesth 1995;15:566-569

6 Delewi R, Zijlstra F, Piek JJ. Left ventricular thrombus formation after acute myocardial infarction. Heart 2012;98(23): $1743-1749$ 